\section{MS12-P6 Best architecture of protein crystal. Database of protein-polymer interactions showing a unique role of PEG in protein crystallization \\ Hašek Jindřich ${ }^{1}$, Skálová Tereza ${ }^{1}$, Kolenko Petr $^{1}$, Dušková Jarmila $^{1}$, Koval Tomáš ${ }^{1}$, Stránský Jan ${ }^{1}$, Švecová Leona ${ }^{1}$, Trundová Marie ${ }^{1}$, Dohnálek Jan ${ }^{1}$}

1. Institute of Biotechnology of Academy of Sciences, BIOCEV, Vestec 25242, Czech Republic

\section{email: hasekjh@seznam.cz}

Protein molecules regularly ordered in crystal remain highly solvated and are continuously in dynamic equilibrium with solution. The stability of molecules in crystal is ensured by the 3D scaffold formed by intermolecular contacts between adhesive patches of neighbor molecules, whereas $30-80 \%$ of crystal content remains dynamically disordered. That is why the stability of the 3D skeleton, i.e. protein crystal architecture (PCA) is so important.

Large surface of protein molecules has usually many adhesive patches and thus we often observe different PCAs of the same protein (polymorphism). Each PCA has its own set of compatible adhesion modes and its own optimal solvent content in crystal. When incompatible adhesion modes are combined during crystal growth, one gets virtually non-diffracting solid phase.

Principle of dominating adhesion mode plays a key role in the control of diffraction quality of crystal. It says that well diffracting crystals grow only when incompatible adhesion modes are suppressed. This can be achieved either by rational composition of crystallization solution using protein surface shielding agents (PSSA) [J.Synchr.Radiation (2011)18,50-52] or by chemical modification of the protein surface, or complexation with high affinity ligands.

Analysis of already solved structures allows planned strengthening of dominant or weakening of non-compatible adhesion modes and a control of the diffraction quality of growing crystals. Theory of protein-protein adhesion modes shows why the poly(ethyleneglycol type polymers (PEGs) are the most successful precipitants [Z.Kristallogr.(2006)23,613-619].

Database of protein-polymers interactions (DPPI) [Z.Kristallogr.(2011)28,475-480] contains about 4000 of experimentally observed PEG-protein interfaces. It consists of a set of protein structures crystallizing with PEG and the script allowing easy visualization of PEG activities on protein surfaces. Seeing the PEG fragments interfering with protein-protein interfaces, with different types of salts, blocking competitive crystal contacts, protein oligomerization, crystallization, or biological activity, helps to understand the dynamic processes during crystal growth and allows rationalization of its performance on molecular basis. DPPI is available from hasekjh@seznam.cz.

The project is supported by BIOCEV CZ.1.05/1.1.00/02.0109 from the ERDF, CSF project 15-15181S of the CSF, grant LG14009 of MSMT, BioStruct-X (EC FP7 project 283570) and Instruct of ESFRI.

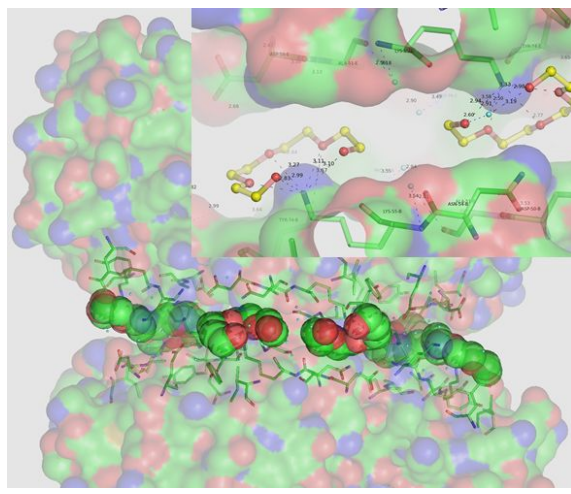

Figure 1. PEG induced adhesion mode as a leading motif for crystallization. The insert shows a typical crown ether conformation of polyether caused by interaction of five oxygens to lysine from one side. It induces strong hydrophobic interaction to the neighbor molecule on the opposite side. PDB code 3NBT.

Keywords: Protein crystal architecture, protein surface shielding agents, protein-protein interfaces, PEG-protein interactions, polymorphism, best diffraction, adhesion mode 